\title{
FACTORES DE RIESGO DE PLACENTA PREVIA EN MUJERES EMBARAZADAS EN UN HOSPITAL DE REPÚBLICA DOMINICANA
}

\author{
Risk factors of placenta previa in pregnant women in a hospital \\ in the Dominican Republic
}

\author{
Laura Manosalvas ${ }^{a}$, Valerie Hernandez ${ }^{b}$, Paola Peñac ${ }^{c}$ Leandro Tapiad, \\ Angiolina Camilo ${ }^{e}$ y Agustín Díaz-Rodríguez ${ }^{f}$
}

Recibido: 25 de marzo, $2020 \bullet$ Aprobado: 6 de junio, 2020

Cómo citar: Manosalvas L, Hernández V, Peña P, Tapia L, Camilo A, Díaz-Rodríguez A. Factores de riesgo de placenta previa en mujeres embarazadas en un hospital de República Dominicana. cysa [Internet]. 17 de septiembre de 2020 [citado 18 de septiembre de 2020];4(3):105-113. Disponible en: https://revistas.intec.edu.do/index.php/cisa/article/view/1910

\section{Resumen}

Introducción: la hemorragia obstétrica es una de las causas de morbilidad y mortalidad materna más común en todo el mundo. La placenta previa es un factor de riesgo importante para la hemorragia obstétrica. El propósito de este estudio es determinar los factores de riesgo relacionados con placenta previa en mujeres embarazadas en Santo Domingo.

Métodos: los casos fueron mujeres cuyos partos fueron complicados con placenta previa y los controles fueron partos sin placenta previa. Se analizaron 16 casos y 76 controles. Se condujo análisis estadístico y se calculó razón de probabilidades (OR).

Resultados: se demostró relación entre placenta previa con antecedente de dos o más abortos con un $\mathrm{OR}=5.3$; con cesárea previa $\mathrm{OR}=3.02$, que aumentaba con más

\footnotetext{
a Escuela de Medicina, Universidad Iberoamericana (UNIBE), Santo Domingo, República Dominicana. ORCID: 0000-0002-1291-7751

Correo-e: Imanosalvas@est.unibe.edu.do

b Escuela de Medicina, Universidad Iberoamericana (UNIBE), Santo Domingo, República Dominicana.

Correo-e: vhernandez7@est.unibe.edu.do

${ }^{\mathrm{c}}$ Instituto de Medicina Tropical \& Salud Global, Universidad Iberoamericana (UNIBE), Santo Domingo, República Dominicana.

Correo-e: ppena9@est.unibe.edu.do
}

\begin{abstract}
Introduction: Obstetric hemorrhage is one of the most common causes of maternal morbidity and mortality worldwide. Placenta previa is an important risk factor for obstetric hemorrhage. The purpose of this study is to determine the risk factors related to placenta previa in pregnant women Santo Domingo.

Methods: The cases were women whose deliveries were complicated with placenta previa and the controls were deliveries without placenta previa. 16 cases and 76 controls were analyzed. Statistical analysis was conducted and odds ratio (OR) was calculated.

Results: The relationship between placenta previa with a history of two or more abortions with an $\mathrm{OR}=5.3$ was demonstrated; with previous caesarean section $\mathrm{OR}=3.02$, which increased with more than 2 caesarean sections with

\footnotetext{
d Escuela de Medicina, Instituto de Medicina Tropical \& Salud Global, Universidad Iberoamericana (UNIBE), Santo Domingo, República Dominicana. Correo-e: 1.tapia@prof.unibe.edu.do

e Escuela de Medicina, Universidad Iberoamericana (UNIBE), Santo Domingo, República Dominicana.

Correo-e: a.camilo@prof.unibe.edu.do

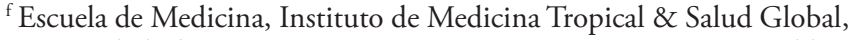
Universidad Iberoamericana (UNIBE), Santo Domingo, República Dominicana. Correo-e: a.diaz38984@unibedom.onmicrosoft.com
} 
de 2 cesáreas con un $\mathrm{OR}=5.91$, con embarazo ectópico previo y antecedente de placenta previa. Las pacientes con placenta previa y antecedente de cirugía tuvieron un mayor riesgo de requerir una histerectomía. Las mujeres con placenta previa y antecedentes de cesáreas múltiples ( 2 o más) tuvieron un mayor riesgo de padecer placenta acreta con un $\mathrm{OR}=18$.

Discusión: nuestro estudio sugiere que abortos múltiples, cesárea previa, múltiples cesáreas anteriores, antecedente de placenta previa y antecedente de embarazo ectópico son factores de riesgo de desarrollar placenta previa.

Palabras claves: placenta previa; cesárea previa; aborto; embarazo ectópico; placenta acreta.

\section{Introducción}

La hemorragia obstétrica es una de las causas de morbilidad y mortalidad materna más común en todo el mundo. ${ }^{1} \mathrm{El} 73 \%$ de las muertes maternas se deben a causas obstétricas directas, siendo la hemorragia obstétrica el $27.1 \%$ de los casos. ${ }^{2}$ Las causas se pueden dividir en tres grupos importantes: placenta previa, placenta abrupta y otras causas. ${ }^{1}$ La placenta previa es un factor de riesgo importante para la hemorragia obstétrica, especialmente en mujeres con cicatriz uterina previa. ${ }^{3}$

La placenta previa es definida como la implantación de la placenta en el segmento uterino inferior, cubriendo o acercado al orificio cervical interno. ${ }^{4}$ Una serie de factores de riesgo han sido descritos como significativos para su desarrollo, sobre todo que es más probable que esté relacionado con afecciones existentes antes del embarazo. ${ }^{5}$ Los factores de riesgo recopilados por el Colegio Real de Obstetras y Ginecólogos (RCOG) son antecedente de placenta previa, parto por cesárea en embarazos previos, aumentando el riesgo a medida que aumentan la cantidad de cesáreas, multiparidad, abortos, edad materna avanzada, estatus de fumadora y concepción asistida. ${ }^{6}$ an $\mathrm{OR}=5.91$, with previous ectopic pregnancy and diagnosis of placenta previa in a previous pregnancy. Patients with placenta previa and a history of surgery had a higher risk of requiring a hysterectomy. Women with placenta previa and a history of multiple caesarean sections ( 2 or more) had a higher risk of suffering from placenta accreta with an $\mathrm{OR}=18$.

Discussion: Our study suggests that multiple abortions, previous caesarean section, multiple previous caesarean sections, antecedents of placenta previa and history of ectopic pregnancy are risk factors of developing placenta previa.

Keywords: Placenta previa; previous cesarean section; abortion; ectopic pregnancy; placenta accreta.

Esta implantación anómala puede llevar al desarrollo de complicaciones y morbilidades tanto en la madre como en el feto. Muchas de estas complicaciones están relacionadas directamente con el sangrado que provoca durante el embarazo. Estas mujeres embarazadas tienen 10 veces más probabilidad de sangrado durante su embarazo que mujeres embarazadas sin esta patología. Otras complicaciones a las que se asocia incluyen histerectomía $(5.3 \%)$, transfusión sanguínea $(21.9 \%)$, presentación anómala del feto (19.8\%), hemorragia postparto $(1.4 \%)$ y placenta acreta $(3 \%) .^{7}$ Las complicaciones y morbilidades fetales se relacionan indirectamente con la prematuridad llevando la mortalidad neonatal a ser tres veces mayor en comparación con las muertes neonatales sin placenta previa. ${ }^{8,9}$ También se asocia a un APGAR menor de 7 después de 1 minuto y 5 minutos (25.3 $\%$, malformaciones congénitas $(11.5 \%)$ y restricción de crecimiento intrauterino $(3.6 \%){ }^{7}$

El propósito de este estudio fue determinar los factores de riesgo relacionados con placenta previa en mujeres embarazadas en el Hospital de la Mujer Dominicana en Santo Domingo, en el periodo enero 2017-noviembre 2019. 


\section{Método}

Se realizó un análisis documental de los expedientes clínicos para poder estudiar y evaluar la información referente a cada paciente. Se establecieron criterios para la inclusión en el estudio dentro un grupo de "Casos" y otro grupo "Control". La selección de la muestra incluyó a las mujeres embarazadas diagnosticadas con placenta previa al momento de su desembarazo, desde enero 2018 hasta noviembre 2019 en el Hospital de la Mujer Dominicana en Santo Domingo, para un total de 16 casos. Por cada caso de placenta previa se seleccionaron los siguientes 4 casos de desembarazo en el Hospital de la Mujer Dominicana en Santo Domingo, en función de controles. Se realizaron análisis estadístico con $\mathrm{IC}=95 \%$ y razón de probabilidades (OR) para establecer y cuantificar la relación entre los factores de riesgo y el resultado final. Se utilizaron los programas STATA y JASP para realizar análisis univariables y multivariables para establecer relación entre variables previamente mencionadas. Se utilizaron los programas STATA y R para establecer análisis de Razón de Probabilidad (Odds Ratio) y los programas Excel y R para organizar en tablas los datos encontrados.

\section{Resultados}

De un total de 6,798 partos durante el periodo de estudio, se encontraron reportados 16 casos de placenta previa y se seleccionaron 76 controles de manera aleatoria y seriada, por cada caso se escogían los siguientes 4 controles. La demografía de este estudio se presenta en la tabla 1.

Se halló que el $68.75 \%$ de las pacientes con placenta previa se encontraba dentro del rango de edad de 25 a 35 años, mientras que el $12.5 \%$ tenía entre 18 y 24 años y el $18.75 \%$ era mayor de 35 ańos. En el grupo control, la mayoría de las mujeres, un 42.11 $\%$ tenía de 25-34 años, un 35.53 \% tenía de 18-24 años y un $17 \%$ era mayor de 35 años.
Se encontró que la gestación media del grupo con placenta previa era de 3.8 mientras que en el grupo control era de 3.08. El antecedente de cantidad de partos vaginales era mayor en el grupo de controles, con una media de 1.06, mientras que en grupo con placenta previa era de 0.81 . El antecedente de cantidad de abortos previos era mayor en el grupo de placenta previa con un 0.81 , en tanto que en el grupo control la media era 0.47 . Entre el grupo de placenta previa se halló que la media de cesáreas anteriores fue de 1.18 en comparación con el grupo control, que fue de 0.55 .

El 56. $25 \%$ de las pacientes con placenta previa eran multíparas y $6.25 \%$ eran grandes multíparas. El grupo control constaba de $42.11 \%$ de multíparas y $7.89 \%$ de grandes multíparas. Se encontró un (1) caso de placenta previa con antecedente de un embarazo ectópico para un $6.25 \%$ en su grupo, a diferencia del grupo de controles, donde no hubo antecedentes de embarazo ectópico.

Se encontró que el $100 \%$ de los partos de mujeres con placenta previa fueron por vía cesárea, mientras que en el grupo control hubo un $57.89 \%$ que fue por vía cesárea y un $42.11 \%$ por vía vaginal.

En relación con los datos del neonato, se identificó que el $62.50 \%$ de los productos de las de las gestantes con placenta previa fue pre-término, mientras que el $37.50 \%$ nació a término, en contraste con el grupo control, donde $17.11 \%$ de los embarazos resultó pre-término frente a $87.50 \%$ que fueron a término.

El $12.50 \%$ de los productos de las pacientes con placenta previa presentó puntuaciones bajas en el APGAR neonatal en 1 minuto, frente a $87.50 \%$ con APGAR normal. Este dato no muestra gran diferencia en contraste con el grupo control, en donde encontramos APGAR neonatal bajo en 1 minuto en $5.26 \%$ de los productos y APGAR normal en $94.74 \%$. Además, se estudió el peso neonatal, en donde encontramos que un $37.50 \%$ 
de los productos de las embarazadas con placenta previa mostró bajo peso, frente a un $62.50 \%$ con peso normal. El grupo control constaba de un $19.74 \%$ de neonatos con peso bajo frente a un $80.26 \%$ con peso adecuado. En cuanto a la talla neonatal, se evidenció que el $31.25 \%$ de los productos de gestantes con placenta previa mostró baja talla, mientras que el $68.75 \%$ tuvo talla normal. En el grupo control encontramos 21.05 $\%$ de los neonatos con talla baja y $78.95 \%$ talla normal.

Entre los datos de complicaciones que se recolectaron para poder analizar relación con los factores de riesgo fueron sangrado pre parto con $62.5 \%$ en el grupo de placenta previa y $1.32 \%$ en el grupo control; sangrado pos parto con un $12.5 \%$ en el grupo de placenta previa y un $2.63 \%$ en el grupo control; histerectomía con $37.5 \%$ en el grupo de placenta previa y $1.32 \%$ en el grupo control; acretismo placentario con $31.25 \%$ en el grupo de placenta previa y ninguno en el grupo control; shock hipovolémico con $18.75 \%$ en el grupo de placenta previa y $1.32 \%$ en el grupo control y presentación anómala con $37.5 \%$ en el grupo de placenta previa y $6.58 \%$ en el grupo control.

Analizamos posibles factores de riesgo para el desarrollo de placenta previa en la población de estudio y controles (tabla 2). No parecía haber una asociación lineal entre placenta previa y antecedente de aborto $(\mathrm{p}=0.46)$, sin embargo, se encontró que hay una relación directa entre placenta previa y antecedente de dos o más abortos con un $\mathrm{OR}=5.3$ (valor de $\mathrm{p}$ de 0.008). El grupo de placenta previa tuvo una totalidad 11 casos de pacientes con antecedente de múltiples abortos mientras que el grupo control tuvo 6 casos. También se demostró que estar expuestas a un procedimiento quirúrgico de tipo cesárea previa en embarazo anteriores era predictivo para placenta previa con un valor de OR $=3.02(\mathrm{p}=0.05) . \mathrm{Y}$, además, se estableció que 2 o más cesáreas previas eran aún más predictivas de desarrollar placenta previa con un $\mathrm{OR}=5.91(\mathrm{p}=0.003)$.
El antecedente de embarazo ectópico mostró relación estadística significativa con la placenta previa, con un (1) caso en el grupo de placenta previa versus ningún caso en el grupo control $(\mathrm{p}<0.05)$. Otra variable estudiada fue el antecedente de haber padecido placenta previa en un embarazo anterior; se encontró un (1) caso en el grupo de placenta previa y ningún caso en el grupo control, pudiendo establecer relación entre historia de placenta previa con el padecimiento de esta anomalía en un subsecuente embarazo $(p=0.02)$. En cuanto a las mujeres estudiadas con placenta previa pudimos ver relación entre algunos factores de riesgo y resultados. Las mujeres con placenta previa y antecedente de gran multiparidad tienen mayor riesgo de padecer sangrado $(\mathrm{p}<0.001)$. Las pacientes con placenta previa y antecedente de cirugía tipo cesárea tuvieron un mayor riesgo de requerir una histerectomía $(\mathrm{p}=0.03)$. Las mujeres con placenta previa y antecedentes de cesáreas múltiples $(2 \mathrm{o}$ más) tuvieron un mayor riesgo de padecer acretismo placentario con un $\mathrm{OR}=18(\mathrm{p}=0.01)$.

\section{Discusión}

El grupo mayoritario de edad en el de placenta previa fue el de 25 a 34 ańos, al igual que en el grupo control, lo cual difiere con la literatura. Sin embargo, para los casos de placenta previa el segundo grupo más numeroso fue el de mayor de 35 años, mientras que en el grupo control fue el de 18 a 24 años. En comparación, el estudio retrospectivo de Vandana intitulado Resultados maternos en placenta previa encontró que fue mayoritario el grupo de edad de 20-29 ańos con un $82 \% .^{10} \mathrm{Al}$ igual que Kollman, quien en su estudio Placenta previa: incidencia, factores de riesgo y resultados establece que la mayoría eran mayores de 25 ańos. ${ }^{11}$ Además, el estudio de Jillani titulado Factor de riesgo para aumento de tasa de placenta previa con cesáreas a repetición, arrojó que la mayoría de las pacientes con placenta previa correspondían a gestantes dentro de los grupos de edades de 31-34 años. ${ }^{12} \mathrm{En}$ cambio, el metaanálisis observacional de Faiz esta- 
blece que la mayoría de los casos de placenta previa eran mayores de 35 ańos, como dicta la mayoría de la literatura. ${ }^{13}$

Ambos grupos tuvieron porcentajes muy similares de frecuencia de paridad, lo que difiere con nuestros antecedentes. Consultando el estudio de Vandana ${ }^{10}$ el $74 \%$ de su población con placenta previa era multípara al igual que el estudio de Jillani, ${ }^{12}$ en donde se encontró que $34.48 \%$ de las gestantes con placenta previa eran multíparas.

En cuanto a la distribución de modalidad de desembarazo, pudimos observar que todas las mujeres con placenta previa fueron desembarazadas por cesárea, presumiblemente por el seguimiento de los protocolos internacionales. Y en el grupo control pudimos ver que la tasa de cesáreas del hospital se asemeja mucho a la tasa de cesáreas nacional de $58.1 \% .{ }^{14}$ Por otro lado, en cuanto al neonato pudimos ver que más de la mitad de las pacientes con placenta previa culminaron en productos pre-termino, mientras que en el grupo control casi su totalidad fue a término. Cabe destacar, como ya se ha mencionado, que este centro sigue recomendaciones internacionales de realizar cesárea a las 36 semanas, la cual pudo alterar los resultados. Asimismo, pudimos observar que los neonatos con puntaje bajo al primer minuto del APGAR del grupo de placenta previa se mantuvieron iguales a los 5 minutos. Pero los neonatos con APGAR bajo del grupo control, la mitad de ellos sí se recuperaron a los 5 minutos.

La placenta previa se asoció con un mayor riesgo general de morbilidad hemorrágica materna con un aumento de sangrado pre-parto y pos parto en el grupo de placenta previa comparado con el grupo control. Se realizaron mayores histerectomías en el grupo de placenta previa que en el grupo control, presumiblemente debido a hemorragia persistente. Estas tuvieron un aumento significativo de shock hipovolémico. Presentaron acretismo placentario cinco mujeres en el grupo de placenta previa en comparación con ninguna mujer del grupo control, siendo este trastorno placentario muy importante, ya que también representa grandes complicaciones.

Los resultados de nuestro estudio indican que abortos múltiples, cirugía anterior tipo cesárea, embarazo ectópico y antecedente de placenta previa son factores de riesgo para el desarrollo de placenta previa. En principio no parecía haber una asociación lineal entre placenta previa y antecedente de aborto, sin embargo, se encontró que hay una relación directa entre placenta previa y antecedente de dos o más abortos con un riesgo aumentado de 5.3 veces. En el estudio de Shobeiri, Determinantes de placenta previa: estudio caso y control, tampoco se pudo establecer relación significativa entre abortos y placenta previa ${ }^{15}$. Mientras que en el estudio de Tuzovic, Factores de Riesgo Obstétricos Asociados al Desarrollo de Placenta Previa, se encontró que existía un riesgo aumentado con un aborto previo de 4.8 veces más y que el riesgo se incrementó con el número creciente de abortos previos. ${ }^{16}$

También se demostró que estar expuestas a un procedimiento quirúrgico de tipo cesárea previa en embarazo anteriores era predictivo para placenta previa, con un valor aumentado de 3.02 veces. Y además se estableció que 2 o más cesáreas previas eran aún más predictivas de desarrollar placenta previa con un riesgo estimado de hasta 5.91 veces más. En el estudio Asociación de parto por cesárea en el primer parto con placenta previa y placenta abrupta en el segundo embarazo, Yang encontró asociación significativa con un riesgo aumentado de 1.47 veces. Esto va contra los hallazgos del estudio de Shobeiri, Determinantes de placenta previa: estudio caso y control, en el que no se encontró relación estadísticamente significativa. ${ }^{15,17}$

Otra variable estudiada fue el antecedente de haber padecido placenta previa en un embarazo anterior. Se encontró un (1) caso en el grupo de placenta previa y ningún caso en el grupo control, pudiendo establecer relación entre historia de placenta previa 
y padecer esta anomalía en un subsecuente embarazo $(\mathrm{p}=0.02)$. En un estudio retrospectivo titulado Factores de riesgo asociados a placenta previa: estudio de casos y controles, Javed encontró relación estadística con un riesgo aumentado aproximado de 6.3 veces más. ${ }^{18}$

Edad materna avanzada, multiparidad, gran multiparidad, embarazo múltiple y trastornos hipertensivos del embarazo no demostraron una relación estadística significativa en nuestro estudio. En cuanto a las mujeres estudiadas con placenta previa pudimos ver relación entre algunos factores de riesgo y resultados. Las mujeres con placenta previa y gran multiparidad tuvieron un mayor riesgo de padecer sangrado. Las pacientes con placenta previa y antecedente de cirugía tipo cesárea tuvieron un mayor riesgo de requerir una histerectomía. Las mujeres con placenta previa y antecedentes de cesáreas múltiples (2 o más) tuvieron un riesgo aumentado de padecer acretismo placentario de hasta 18 veces más, lo cual coincide con la revisión de Dulay: Placenta Previa, en la que plantea que el riesgo de acretismo placentario aumenta según el número de partos por cesárea previos (de alrededor del $10 \%$ si han tenido un parto por cesárea a $>60 \%$ si han tenido $>4) .{ }^{19}$

\section{Consideraciones éticas}

El estudio fue realizado con la aprobación del Comité de Ética Institucional de la Universidad Iberoamericana (UNIBE) para llevarlo a cabo bajo en número: DIA-IRB00918-2019

\section{Bibliografía}

1. Naiknaware S. Antepartum Haemorrhage: Causes \& Its Effects on Mother and Child: An Evaluation. Obstetrics \& Gynecology International Journal. 2015;3(1).

2. Say L, Chou D, Gemmill A, Tunçalp Ö, Moller A, Daniels J et al. Global causes of maternal death: a WHO systematic analysis. The Lancet Global Health. 2014;2(6):e323-e333.

3. Getahun D, Oyelesse Y, Salihu HM, Ananth CV. Previous Caesarean Delivery and Risks Of Placenta PreviaAnd Abruption. Obstet Gynecol., 2006;107:771-8. Available from: https://www. ncbi.nlm.nih.gov/pubmed/16582111

4. Cunningham F, Leveno K, Bloom S, Spong CY, Dashe J. Williams obstetrics, 24e. McGrawhill; 2014.

5. Yang Q, Wen SW, Phillips K, Oppenheimer L, Black D, Walker MC. Comparison of maternal risk factors between placental abruption and placenta previa. Am J Perinatol 2009;26:27986.

6. RCOG. Antepartum Haemorrhage [Internet]. Guidelines Committee of the Royal College of Obstetricians and Gynaecologists; 2011 Available from: https://www.rcog.org.uk/globalassets/documents/guidelines/gtg_63.pdf [citado el 11 diciembre 2019].

7. Rosenberg T, Pariente G, Sergienko R, Wiznitzer A, Sheiner E. Critical analysis of risk factors and outcome of placenta previa. Archives of Gynecology and Obstetrics [Internet]. 2011;284(1):47-51. Available from: https://www.scopus.com/record/display.uri?ei$\mathrm{d}=2$-s2.0-79958824109\&origin=inward \&txGid=f7f6f5dad6f18087697db1fdf81f26b9

8. Ananth CV, Demisse K, Smulian JC, Vintzileos AM. Placenta previa in singleton and twin births in the United States, 1989 through 1998: a comparison of risk factor profiles and associated conditions. Am J Obstet Gynecol. 2003;188:275-81 Available from: https://www. ncbi.nlm.nih.gov/pubmed/12548229 
9. Salihu HM, Li Q, Rouse DJ, Alexander GR. Placenta previa: Neonatal death after live births in the United States. American Journal of Obstetrics and Gynecology [Internet]. 2003;188(5):1305-9. Available from: https:// www.sciencedirect.com/science/article/pii/ S0002937803001339

10. Vandana RG, Neelima K, Madhuri G, Varalakshmi P. Maternal outcome in placenta previa - A retrospective study. International Archives of Integrated Medicine [Internet]. 2019;6(3):143-7. Available from: http:// search.ebscohost.com/login.aspx?direct=true \&db=a9h\&AN=135810318\&lang=es\&site=ehost-live [citado 27 de abril 2019]

11. Kollmann M, Gaulhofer J, Lang U, Klaritsch P. Placenta praevia: incidence, risk factors and outcome. The Journal of Maternal-Fetal \& Neonatal Medicine. 2015;29(9):1395-8. Available from: https://www.thieme-connect.com/ products/ejournals/abstract/10.1055/s-00331354833

12. Jillani K, Shaikh F, Siddiqui SM, Siddiqui MA. Repeated Cesarean Sections: A Risk Factor for Rising Rate of Placenta Previa. Medical Channel [Internet]. 2010;16(3):409-12. Available from: http://search.ebscohost.com/login.aspx?direc$\mathrm{t}=\mathrm{true} \& \mathrm{db}=\mathrm{a} 9 \mathrm{~h} \& A \mathrm{AN}=55121100 \&$ lang $=\mathrm{es} \&$ site=ehost-live [citado 22 de marzo 2019]

13. Faiz AS, Ananth CV. Etiology and risk factors for placenta previa: An overview and metaanalysis of observational studies. Journal of Maternal-Fetal and Neonatal Medicine [Internet]. 2003;13(3):175-90. Available from: https://www.scopus.com/record/display.uri?ei$\mathrm{d}=2$-s2.0-0037353124\&origin=inward\&txGi$\mathrm{d}=\mathrm{b} 4 \mathrm{f} 31 \mathrm{e} 853 \mathrm{~d} 4 \mathrm{dce} 1278 \mathrm{f} 2 \mathrm{e} 0 \mathrm{a} 307 \mathrm{ba} 4500$

14. Oficina Nacional de Estadística y UNICEF, 2016. Encuesta Nacional de Hogares de Propósitos Múltiples - Encuesta de Indicadores Múltiples por Conglomerados 2014, Informe Final.
[Internet]. Santo Domingo, República Dominicana: Oficina Nacional de Estadística. Available from: https://www.one.gob.do/encuestas/ enhogar/enhogar-2014

15. Shobeiri F, Jenabi E, Karami M, Karimi S. Determinants of placenta previa: a case-control study. Biomedical Research and Therapy. 2017;4(06):1411-9. Available from: https:// doi.org/10.15419/bmrat.v4i06.182

16. Tuzović L, Djelmis J, Ilijc M. Obstetric risk factors associated with placenta previa development: case-control study. -PubMed- NCBI [Internet]. Ncbi.nlm.nih.gov. 2003. Disponible en: https://www.ncbi.nlm.nih.gov/pubmed/?term $=14652887$ [citado 9 de diciembre 2019]

17. Yang Q, Wen S, Oppenheimer L, Chen X, Black D, Gao J et al. Association of caesarean delivery for first birth with placenta praevia and placental abruption in second pregnancy. BJOG: An International Journal of Obstetrics \& Gynaecology. 2007;114(5):609-13. Available from: https://obgyn.onlinelibrary.wiley.com/ doi/full/10.1111/j.1471-0528.2007.01295.x

18. Latif L, Javed Iqbal U, Aftab M.U. Associated risk factors of placenta previa a matched case control study. Pakistan Journal of Medical and Health Sciences. 2015;9:1344-6. Available from: https://www.researchgate.net/ publication/298640837_Associated_risk_ factors_of_placenta_previa_a_matched_case_ control_study

19. Dulay A. Placenta previa - Ginecología y obstetricia - Manual MSD versión para profesionales [Internet]. Manual MSD versión para profesionales; 2017. Available from: https:// www.msdmanuals.com/es/professional/gineco$\log \% \mathrm{C} 3 \% \mathrm{ADa}$-y-obstetricia/anomal\%C $3 \% \mathrm{~A}-$ Das-del-embarazo/placenta-previa [citado 12 de abril 2019]. 


\section{Anexos}

Tabla 1. Datos Generales de las pacientes estudiadas en el Hospital de la Mujer Dominicana, en el periodo enero 2018 - noviembre 2019 (N=92)

\begin{tabular}{|c|c|c|c|c|}
\hline & \multicolumn{2}{|c|}{ Diagnóstico Placenta Previa n=16 (17.4\%) } & \multicolumn{2}{|c|}{ Controles $n=76(82.6 \%)$} \\
\hline & $\mathrm{n}$ & $\%$ & $\mathrm{n}$ & $\%$ \\
\hline \multicolumn{5}{|l|}{ Grupo de Edades } \\
\hline $18-24$ & 2 & 12.50 & 27 & 35.53 \\
\hline $25-34$ & 11 & 68.75 & 32 & 42.11 \\
\hline$>35$ & 3 & 18.75 & 17 & 22.37 \\
\hline \multicolumn{5}{|l|}{ Datos Embarazo } \\
\hline Media Gestación & 3.875 & IC: $95 \%$ & 3.08 & IC: $95 \%$ \\
\hline Media Para & 0.813 & IC: $95 \%$ & 1.065 & IC: $95 \%$ \\
\hline Media Abortos & 0.813 & IC: $95 \%$ & 0.473 & IC: $95 \%$ \\
\hline Media Cesáreas & 1.186 & IC: $95 \%$ & 0.553 & IC: $95 \%$ \\
\hline Multiparidad & 9 & 56.25 & 32 & 42.11 \\
\hline Gran Multiparidad & 1 & 6.25 & 6 & 7.89 \\
\hline Embarazo Ectópico & 1 & 6.25 & 0 & 0 \\
\hline Parto por Cesárea & 16 & 100 & 44 & 57.89 \\
\hline Parto Vaginal & 0 & 0 & 32 & 42.11 \\
\hline \multicolumn{5}{|l|}{ Datos del Neonato } \\
\hline \multicolumn{5}{|l|}{ Edad Gestacional } \\
\hline Pre-término & 10 & $62.50 \%$ & 13 & $17.11 \%$ \\
\hline Término & 6 & $37.50 \%$ & 63 & $82.89 \%$ \\
\hline \multicolumn{5}{|c|}{ APGAR Neonatal 1- Minuto } \\
\hline Bajo & 2 & $12.50 \%$ & 4 & $5.26 \%$ \\
\hline Normal & 14 & $87.50 \%$ & 72 & $94.74 \%$ \\
\hline \multicolumn{5}{|c|}{ APGAR Neonatal 5- Minuto } \\
\hline Bajo & 2 & $12.50 \%$ & 2 & $2.63 \%$ \\
\hline Normal & 14 & $87.50 \%$ & 74 & $97.37 \%$ \\
\hline \multicolumn{5}{|l|}{ Peso Neonatal } \\
\hline Bajo & 6 & $37.50 \%$ & 15 & $19.74 \%$ \\
\hline Normal & 10 & $62.50 \%$ & 61 & $80.26 \%$ \\
\hline \multicolumn{5}{|l|}{ Talla Neonatal } \\
\hline Bajo & 5 & $31.25 \%$ & 16 & $21.05 \%$ \\
\hline Normal & 11 & $68.75 \%$ & 60 & $78.95 \%$ \\
\hline \multicolumn{5}{|l|}{ Complicaciones } \\
\hline \multicolumn{5}{|l|}{ Sangrado } \\
\hline Sangrado Pre Parto & 10 & 62.5 & 1 & 1.32 \\
\hline Sangrado Pos Parto & 2 & 12.5 & 2 & 2.63 \\
\hline Histerectomía & 6 & 37.5 & 1 & 1.32 \\
\hline Placenta Acreta & 5 & 31.25 & 0 & 0 \\
\hline Shock Hipovolémico & 3 & 18.75 & 1 & 1.32 \\
\hline Presentación Anómala & 6 & 37.5 & 5 & 6.58 \\
\hline
\end{tabular}

Fuente: elaboración propia. 
Tabla 2. Análisis Multifactorial de Potenciales Factores de Riesgo para el desarrollo de Placenta Previa en Placenta Previa y Controles en el Hospital de la Mujer Dominicana, en el periodo enero 2018 - noviembre 2019

\begin{tabular}{|c|c|c|c|c|}
\hline \multirow[b]{2}{*}{ Factores de Riesgo } & \multicolumn{2}{|c|}{ Grupo de Estudio } & \multicolumn{2}{|c|}{ Análisis Estadístico } \\
\hline & Placenta Previa & Controles & Odds Ratio & Valor P \\
\hline \multicolumn{5}{|l|}{$>35$ años } \\
\hline$>35$ & 3 & 17 & \multirow{2}{*}{1.248587571} & \multirow{2}{*}{0.749777822} \\
\hline $18-34$ & 13 & 59 & & \\
\hline \multicolumn{5}{|l|}{ Multiparidad } \\
\hline Sí & 9 & 32 & \multirow{2}{*}{1.767857143} & \multirow{2}{*}{0.300848984} \\
\hline No & 7 & 44 & & \\
\hline \multicolumn{5}{|l|}{ Gran Multiparidad } \\
\hline Sí & 1 & 6 & \multirow{2}{*}{1.285714282} & \multirow{2}{*}{0.821569285} \\
\hline No & 15 & 70 & & \\
\hline \multicolumn{5}{|l|}{ Aborto } \\
\hline Sí & 9 & 50 & \multirow{2}{*}{1.495726493} & \multirow{2}{*}{0.469614585} \\
\hline No & 7 & 26 & & \\
\hline \multicolumn{5}{|l|}{ Abortos Múltiples } \\
\hline Sí & 11 & 70 & \multirow{2}{*}{5.303030291} & \multirow{2}{*}{0.008870181} \\
\hline No & 5 & 6 & & \\
\hline \multicolumn{5}{|l|}{ Cesárea Anterior } \\
\hline Sí & 11 & 32 & \multirow{2}{*}{3.025} & \multirow{2}{*}{0.052196319} \\
\hline No & 5 & 44 & & \\
\hline \multicolumn{5}{|l|}{ Embarazo Ectópico } \\
\hline Sí & 1 & 0 & \multirow{2}{*}{ N/A } & \multirow{2}{*}{0.028423456} \\
\hline No & 15 & 76 & & \\
\hline \multicolumn{5}{|l|}{ Embarazo Múltiple } \\
\hline Sí & 1 & 2 & \multirow{2}{*}{0.405405405} & \multirow{2}{*}{0.458894156} \\
\hline No & 15 & 74 & & \\
\hline \multicolumn{5}{|l|}{ Múltiple Cesárea } \\
\hline Sí & 10 & 69 & \multirow{2}{*}{5.914285714} & 0.0031514 \\
\hline No & 6 & 7 & & \\
\hline HTA & & & & \\
\hline Sí & 5 & 19 & 0.73333333 & 060482188 \\
\hline No & 11 & 57 & & 0.00483188 \\
\hline $\begin{array}{l}\text { Antecedentes Placenta } \\
\text { Previa }\end{array}$ & & & & \\
\hline Sí & 1 & 0 & N/A & 0,028423456 \\
\hline No & 15 & 76 & & \\
\hline
\end{tabular}

Fuente: elaboración propia. 